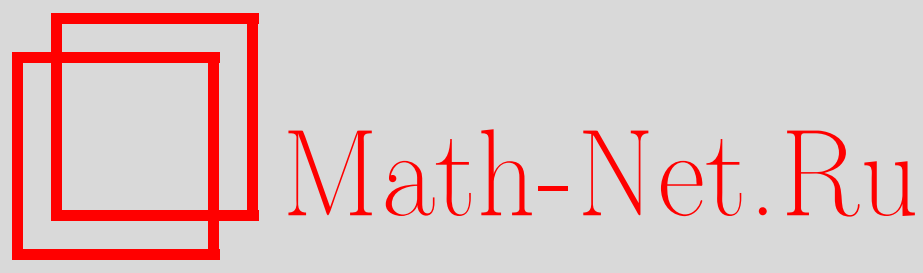

Б. Б. Маркив, И. П. Омелян, М. В. Токарчук, Неравновесный статистический оператор в обобщенной молекулярной гидродинамике жидкостей, ТМФ, 2008, том 154, номер 1, 91-101

DOI: https://doi.org/10.4213/tmf6153

Использование Общероссийского математического портала Math-Net.Ru подразумевает, что вы прочитали и согласны с пользовательским соглашением http: //www . mathnet.ru/rus/agreement

Параметры загрузки:

IP : 34.239 .49 .27

26 апреля 2023 г., 08:59:15

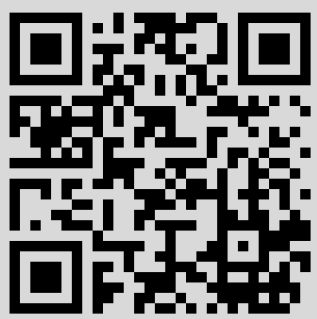




\title{
НЕРАВНОВЕСНЫЙ СТАТИСТИЧЕСКИЙ ОПЕРАТОР В ОБОБЩЕННОЙ МОЛЕКУЛЯРНОЙ ГИДРОДИНАМИКЕ ЖИДКОСТЕЙ
}

\begin{abstract}
Обсуждается важная роль метода неравновесного статистического оператора Зубарева в обобщенной молекулярной гидродинамике жидкостей. Использование этого метода дает возможность развить последовательный подход обобщенных коллективных возбуждений для простых, ионных, полярных, магнитных и других жидкостей. Получен неравновесный статистический оператор и соответствующие уравнения переноса для системы, релаксирующей к состоянию молекулярной гидродинамики.
\end{abstract}

Ключевые слова: неравновесный статистический оператор, временны́е корреляционные функции, коэффициенты переноса, оператор Лиувилля.

\section{1. ВВЕДЕНИЕ}

Важным достижением современной неравновесной теории жидкостей являются результаты теории молекулярной гидродинамики [1]-[12]. На ее основе исследовались коллективные моды, временны́е корреляционные функции, обобщенные коэффициенты переноса для модели Леннарда-Джонса простых жидкостей [1], [2], [8], [12]. Методом неравновесного статистического оператора Зубарева [13]-[16] и методом проекционных операторов [17] была построена обобщенная молекулярная гидродинамика на основе расширения параметров сокращенного описания, которые лежат в основе обобщенного подхода коллективных возбуждений [12], [18]-[20]. В отличие от метода проекционных операторов, в методе неравновесного статистического оператора проекционные операторы возникают естественным образом вследствие исключения производных термодинамических параметров по времени, сопряженных средним значениям параметров сокращенного описания. Такой подход показал, что, кроме известных гидродинамических мод, существуют "кинетические" моды, которые дают существенный вклад в области промежуточных значений волновых векторов и частот для коллективных возбуждений. Он также дал возможность исследовать спектр коллективных возбуждений, временнь́е корреляционные функции и

* Институт физики конденсированных систем Национальной академии наук Украины, Львов, Украина. E-mail: markiv@icmp.lviv.ua; omelyan@icmp.lviv.ua; mtok@icmp.lviv.ua 
обобщенные коэффициенты переноса для магнитных [21]-[26], полярных [27]-[32], ионных [16], [33]-[35] жидкостей, полуквантового гелия [36], [37], бинарных смесей [38]-[40], металлических сплавов [41]-[46]. Обобщенное описание кинетических и гидродинамических процессов в теории плотных газов и жидкостей было предложено в работах [47]-[49]. В этом подходе в основном исследовались обобщенные кинетические уравнения для плотных газов и плазмы [50]-[53], а переход к состоянию молекулярной гидродинамики анализировался для простых жидкостей в работе [49] и для квантовых бозе-систем в работе [54].

\section{2. НЕРАВНОВЕСНЫЙ СТАТИСТИЧЕСКИЙ ОПЕРАТОР И УРАВНЕНИЯ ОБОБЩЕННОЙ ГИДРОДИНАМИКИ}

В основе молекулярной гидродинамики пространственно-однородных простых жидкостей лежат уравнения переноса для средних $\left\langle\tilde{a}_{\vec{k}}\right\rangle^{t}=\left(\left\langle\hat{n}_{\vec{k}}\right\rangle^{t},\left\langle\hat{\vec{\jmath}}_{\vec{k}}\right\rangle^{t},\left\langle\hat{h}_{\vec{k}}\right\rangle^{t}\right)$, где $\hat{n}_{\vec{k}}=\sum_{j=1}^{N} e^{-i \vec{k} \vec{r}_{j}}, \hat{\vec{j}}_{\vec{k}}=\sum_{j=1}^{N} \vec{p}_{j} e^{-i \vec{k} \vec{r}_{j}}, \hat{h}_{\vec{k}}=\widehat{E}_{\vec{k}}-\left\langle\widehat{E}_{\vec{k}} \hat{n}_{-\vec{k}}\right\rangle_{0}\left\langle\hat{n}_{\vec{k}} \hat{n}_{-\vec{k}}\right\rangle_{0}^{-1} \hat{n}_{\vec{k}}-$ фурье-компоненты плотности числа частиц, плотности импульса и плотности обобщенной энтальпии, соответственно [1], [10], [11];

$$
\widehat{E}_{\vec{k}}=\sum_{j=1}^{N}\left(\frac{p_{j}^{2}}{2 m}+\frac{1}{2} \sum_{l \neq j=1}^{N} \Phi\left(r_{l j}\right)\right) e^{-i \vec{k} \vec{r}_{j}}
$$

- фурье-компоненты плотности полной энергии. Здесь $\Phi\left(r_{l j}\right)$ - парный потенциал взаимодействия частиц массы $m$, полное число которых равно $N$, с координатами фазового пространства $\left(\vec{p}_{j}, \vec{r}_{j}\right)$ - радиус-векторами импульса и пространственной координаты, $r_{l j}$ - расстояние между частицами, $\vec{k}$ - волновой вектор. Такие уравнения переноса имеют следующую структуру [1], [10], [11]:

$$
\frac{\partial}{\partial t}\left\langle\hat{a}_{l, \vec{k}}\right\rangle^{t}-\sum_{m} i \Omega_{l m}^{\mathrm{H}}(\vec{k})\left\langle\hat{a}_{m, \vec{k}}\right\rangle^{t}+\sum_{m} \int_{-\infty}^{t} e^{\varepsilon\left(t^{\prime}-t\right)} \varphi_{l m}^{\mathrm{H}}\left(\vec{k}, t, t^{\prime}\right)\left\langle\hat{a}_{m, \vec{k}}\right\rangle^{t^{\prime}} d t^{\prime}=0,
$$

где введены обозначения $l=1,2,3, \hat{a}_{1, \vec{k}}=\hat{n}_{\vec{k}}, \hat{a}_{2, \vec{k}}=\hat{\vec{\jmath}}_{\vec{k}}, \hat{a}_{3, \vec{k}}=\hat{h}_{\vec{k}}$. В этом уравнении $i \Omega_{m l}^{\mathrm{H}}(\vec{k})=\sum_{l^{\prime}}\left\langle\dot{\hat{a}}_{m, \vec{k}} \hat{a}_{l^{\prime},-\vec{k}}\right\rangle_{0} \widetilde{\Phi}_{l^{\prime} l}^{-1}(\vec{k})$ - элементы матрицы

$$
i \widetilde{\Omega}^{\mathrm{H}}(\vec{k})=\left(\begin{array}{ccc}
0 & i \Omega_{n j} & 0 \\
i \Omega_{j n} & 0 & i \Omega_{j h} \\
0 & i \Omega_{h j} & 0
\end{array}\right)_{(\vec{k})}
$$

которые описывают недиссипативные процессы и представляют собой нормированные равновесные корреляционные функции

$$
\begin{aligned}
& i \Omega_{n j}(\vec{k})=\left\langle\dot{\hat{n}}_{\vec{k}} \hat{\vec{\jmath}}_{-\vec{k}}\right\rangle_{0}\left\langle\hat{\vec{\jmath}}_{\vec{k}} \cdot \hat{\vec{J}}_{-\vec{k}}\right\rangle_{0}^{-1}, \\
& i \Omega_{j n}(\vec{k})=\left\langle\dot{\overrightarrow{\vec{J}_{k}}} \hat{n}_{-\vec{k}}\right\rangle_{0}\left\langle\hat{n}_{\vec{k}} \hat{n}_{-\vec{k}}\right\rangle_{0}^{-1}, \\
& i \Omega_{h j}(\vec{k})=\left\langle\dot{\hat{h}}_{\vec{k}} \hat{\vec{\jmath}}_{-\vec{k}}\right\rangle_{0}\left\langle\hat{\vec{j}}_{\vec{k}} \cdot \hat{\vec{\jmath}}_{-\vec{k}}\right\rangle_{0}^{-1}, \\
& i \Omega_{j h}(\vec{k})=\left\langle\dot{\overrightarrow{\vec{j}_{k}}} \hat{h}_{-\vec{k}}\right\rangle_{0}\left\langle\hat{h}_{\vec{k}} \hat{h}_{-\vec{k}}\right\rangle_{0}^{-1} \text {; }
\end{aligned}
$$


$\widetilde{\Phi}_{l l^{\prime}}^{-1}(\vec{k})$ - элементы матрицы, обратной к матрице $\widetilde{\Phi}(\vec{k})$ :

$$
\widetilde{\Phi}(\vec{k})=\left(\begin{array}{ccc}
\left\langle\hat{n}_{\vec{k}} \hat{n}_{-\vec{k}}\right\rangle_{0} & 0 & 0 \\
0 & \left\langle\hat{\vec{j}}_{\vec{k}} \cdot \hat{\vec{\jmath}}_{-\vec{k}}\right\rangle_{0} & 0 \\
0 & 0 & \left\langle\hat{h}_{\vec{k}} \hat{h}_{-\vec{k}}\right\rangle_{0}
\end{array}\right),
$$

структура которой указывает на ортогональность переменных $\hat{n}_{\vec{k}}, \hat{\vec{\jmath}}_{\vec{k}}, \hat{h}_{\vec{k}}$ в смысле средних $\left\langle\hat{n}_{\vec{k}} \hat{\vec{J}}_{-\vec{k}}\right\rangle_{0}=0,\left\langle\hat{n}_{\vec{k}} \hat{h}_{-\vec{k}}\right\rangle_{0}=0,\left\langle\hat{\vec{j}}_{\vec{k}} \hat{h}_{-\vec{k}}\right\rangle_{0}=0$. При этом $\left\langle\hat{n}_{\vec{k}} \hat{n}_{-\vec{k}}\right\rangle_{0}=S_{2}(\vec{k})-$ статический структурный фактор атомов простой жидкости, где усреднение $\langle\ldots\rangle_{0}=$ $\int d \Gamma_{N} \ldots \varrho_{0}\left(x^{N}\right)$ выполняется с равновесным статистическим оператором $\varrho_{0}\left(x^{N}\right)=$ $Z^{-1} e^{-\beta(H-\mu N)}, Z=\int d \Gamma_{N} e^{-\beta(H-\mu N)}-$ большая статистическая сумма, $\beta=1 / k_{\mathrm{b}} T$, $k_{\mathrm{Б}}$ - постоянная Больцмана, $T$ - равновесное значение температуры, $\mu$ - химический потенциал, $H=\sum_{j=1}^{N}\left(p_{j}^{2} /(2 m)+(1 / 2) \sum_{l \neq j=1}^{N} \Phi\left(r_{l j}\right)\right)$ - гамильтониан атомов простой жидкости. Диссипативные процессы в уравнениях молекулярной гидродинамики (1) описываются ядрами переноса

$$
\varphi_{l m}^{\mathrm{H}}\left(\vec{k} ; t, t^{\prime}\right)=\sum_{l^{\prime}}\left\langle I_{l}^{\mathrm{H}}(\vec{k}) T_{0}^{\mathrm{H}}\left(t, t^{\prime}\right) I_{l^{\prime}}^{\mathrm{H}}(-\vec{k})\right\rangle_{0} \widetilde{\Phi}_{l^{\prime} m}^{-1}(\vec{k}),
$$

которые являются элементами матрицы функций памяти:

$$
\widetilde{\varphi}^{\mathrm{H}}\left(\vec{k} ; t, t^{\prime}\right)=\left(\begin{array}{ccc}
0 & 0 & 0 \\
0 & \varphi_{j j}^{\mathrm{H}} & \varphi_{j h}^{\mathrm{H}} \\
0 & \varphi_{h j}^{\mathrm{H}} & \varphi_{h h}^{\mathrm{H}}
\end{array}\right)_{\left(\vec{k} ; t, t^{\prime}\right)},
$$

где $I_{j}^{\mathrm{H}}(\vec{k})=\left(1-P_{\mathrm{H}}\right) i L_{N} \hat{\vec{\jmath}}_{\vec{k}}=-i \vec{k}: \stackrel{\leftrightarrow}{\pi}_{\vec{k}}, I_{h}^{\mathrm{H}}(\vec{k})=\left(1-P_{\mathrm{H}}\right) i L_{N} \hat{h}_{\vec{k}}=-i \vec{k} \cdot \hat{\vec{q}}_{\vec{k}}-$ обобщенные потоки, связанные соответственно с $\hat{\pi}_{\vec{k}}, \hat{\vec{q}}_{\vec{k}}-$ фурье-компонентами обобщенных тензора вязкости и потока энтальпии;

$$
i L_{N}=\sum_{j=1}^{N} \frac{\vec{p}_{j}}{m} \frac{\partial}{\partial \vec{r}_{j}}-\frac{1}{2} \sum_{l \neq j=1}^{N} \frac{\partial}{\partial \vec{r}_{j}} \Phi\left(r_{l j}\right)\left(\frac{\partial}{\partial \vec{p}_{j}}-\frac{\partial}{\partial \vec{p}_{l}}\right)
$$

- оператор Лиувилля, соответствующий гамильтониану $H$ системы; $P_{\mathrm{H}}$ - проекционный оператор Мори, построенный на гидродинамических ортогональных переменных $\hat{n}_{\vec{k}}, \hat{\vec{\jmath}}_{\vec{k}}, \hat{h}_{\vec{k}} ; T_{0}^{\mathrm{H}}\left(t, t^{\prime}\right)=e^{\left(t^{\prime}-t\right)\left(1-P_{\mathrm{H}}\right) i L_{N}}-$ оператор эволюции с учетом проектирования Мори на пространство ортогональных динамических переменных $\hat{n}_{\vec{k}}$, $\hat{\vec{\jmath}}_{\vec{k}}, \hat{h}_{\vec{k}}$ [1], [11], [15]. В формуле (9) учтено, что $I_{n}^{\mathrm{H}}(\vec{k})=\left(1-P_{\mathrm{H}}\right) i L_{N} \hat{n}_{\vec{k}}=0$. Ядра переноса матрицы (9) в представлении Лапласа $\left(A(z)=i \int_{0}^{\infty} d t e^{i z t} A(t), z=\omega+i \varepsilon\right.$, $\varepsilon \rightarrow+0)$ определяют обобщенные коэффициенты вязкости $\eta(\vec{k}, z)$, теплопроводности $\lambda(\vec{k}, z)$ и перекрестные коэффициенты переноса $\xi(\vec{k}, z)[5],[6],[10],[11]$ :

$$
\begin{aligned}
\varphi_{j j}^{\mathrm{H}}(\vec{k}, z) & =i k^{2} \eta(\vec{k}, z) \frac{\beta}{m n}, \\
\varphi_{h h}^{\mathrm{H}}(\vec{k}, z) & =i k^{2} \lambda(\vec{k}, z) \frac{k_{\mathrm{5}} \beta^{2}}{c_{v}(\vec{k})}, \\
\varphi_{j h}^{\mathrm{H}}(\vec{k}, z) & =i k^{2} \xi(\vec{k}, z) \frac{k_{\mathrm{5}} \beta^{2}}{c_{v}(\vec{k})},
\end{aligned}
$$


где $c_{v}(\vec{k})$ - обобщенная теплоемкость простых жидкостей. Уравнения молекулярной гидродинамики (1) также определяют временны́е корреляционные функции гидродинамических переменных $\hat{n}_{\vec{k}}, \hat{\vec{j}}_{\vec{k}}, \hat{h}_{\vec{k}}[10],[11]$ :

$$
\frac{\partial}{\partial t} \widetilde{\Phi}^{\mathrm{H}}(\vec{k} ; t)-i \widetilde{\Omega}^{\mathrm{H}}(\vec{k}) \widetilde{\Phi}^{\mathrm{H}}(\vec{k} ; t)+\int_{-\infty}^{t} e^{\varepsilon\left(t^{\prime}-t\right)} \widetilde{\varphi}^{\mathrm{H}}\left(\vec{k}, t, t^{\prime}\right) \widetilde{\Phi}^{\mathrm{H}}\left(\vec{k} ; t^{\prime}\right) d t^{\prime}=0
$$

где

$$
\widetilde{\Phi}(\vec{k} ; t)=\left(\begin{array}{lll}
\Phi_{n n} & \Phi_{n j} & \Phi_{n h} \\
\Phi_{j n} & \Phi_{j j} & \Phi_{j h} \\
\Phi_{h n} & \Phi_{h j} & \Phi_{h h}
\end{array}\right)_{(\vec{k} ; t)}
$$

- матрица временнь́х корреляционных функций $\Phi_{l m}(\vec{k} ; t)=\left\langle\hat{a}_{l, \vec{k}}(t) \hat{a}_{m,-\vec{k}}(0)\right\rangle_{0}$ гидродинамических переменных. В рамках метода неравновесного статистического оператора Зубарева уравнения молекулярной гидродинамики (1) получены с помощью неравновесного статистического оператора [10], [11]:

$$
\begin{aligned}
\varrho^{\mathrm{H}}\left(x^{N} ; t\right)=( & +\sum_{l, m} \sum_{\vec{k}}\left(\hat{a}_{l,-\vec{k}} \widetilde{\Phi}_{l m}^{-1}(\vec{k})\left\langle\hat{a}_{m, \vec{k}}\right\rangle^{t}-\right. \\
& \left.\left.-\int_{-\infty}^{t} e^{\varepsilon\left(t^{\prime}-t\right)} T_{0}^{\mathrm{H}}\left(t, t^{\prime}\right) I_{l}^{\mathrm{H}}(-\vec{k}) \widetilde{\Phi}_{l m}^{-1}(\vec{k})\left\langle\hat{a}_{m, \vec{k}}\right\rangle^{t^{\prime}} d t^{\prime}\right)\right) \varrho_{0}\left(x^{N}\right),
\end{aligned}
$$

т.е. состоянию молекулярной гидродинамики простой жидкости отвечает неравновесный статистический оператор (12). Он является функцией расширенного набора динамических переменных $\hat{n}_{\vec{k}}, \hat{\vec{\jmath}}_{\vec{k}}, \hat{h}_{\vec{k}}$ и обобщенных потоков $I_{n}^{\mathrm{H}}(\vec{k})=0, I_{j}^{\mathrm{H}}(\vec{k})$, $I_{h}^{\mathrm{H}}(\vec{k})$, которые определяют обобщенные коэффициенты переноса (9). Набор переменных $\hat{a}_{l, \vec{k}}, I_{l}^{\mathrm{H}}(\vec{k})$ стал основой построения обобщенных уравнений гидродинамики для простых жидкостей [8], [12], [17]-[20], бинарних смесей [18], [38], [55], ионных жидкостей [16], [33]-[35], полуквантового гелия [36], [37], квантовых бозе-систем [56]-[58], в методе функций Грина [9] и др. Такой подход дал возможность в рамках одной схемы проводить исследования временнь́х корреляционных функций “плотность-плотность" (динамического структурного фактора), “поток-поток”, “энергия-энергия”, их перекрестных корреляционных функций, а также обобщенных коэффициентов переноса (10), включая спектр кинетических и гидродинамических мод. Такому расширенному описанию гидродинамического состояния системы также соответствует неравновесный статистический оператор, построенный для простых жидкостей, ионных систем и др. [16], [59]-[61].

Для простой жидкости неравновесный статистический оператор обобщенной гидродинамики имеет следующую структуру, подобную (12):

$$
\begin{aligned}
\varrho^{\mathrm{GH}}\left(x^{N} ; t\right)=( & +\sum_{l, m} \sum_{\vec{k}}\left(\widehat{Y}_{l,-\vec{k}}\left(\widetilde{\Phi}^{\mathrm{G}}\right)_{l m}^{-1}(\vec{k})\left\langle\widehat{Y}_{m, \vec{k}}\right\rangle^{t}-\right. \\
& \left.\left.-\int_{-\infty}^{t} e^{\varepsilon\left(t^{\prime}-t\right)} T_{0}^{\mathrm{GH}}\left(t, t^{\prime}\right) I_{l}^{\mathrm{GH}}(-\vec{k})\left(\widetilde{\Phi}^{\mathrm{G}}\right)_{l m}^{-1}(\vec{k})\left\langle\widehat{Y}_{m, \vec{k}}\right\rangle^{t^{\prime}} d t^{\prime}\right)\right) \varrho_{0}\left(x^{N}\right),
\end{aligned}
$$


где $\widehat{Y}_{l, \vec{k}}=\left\{\hat{a}_{l, \vec{k}}, \hat{\leftrightarrow}_{\vec{k}}, \hat{\vec{q}}_{\vec{k}}\right\}$ - расширенный набор динамических переменных, которые являются ортогональными, образуя диагональную матрицу равновесных корреляционных функций

$$
\widetilde{\Phi}(\vec{k})=\left(\begin{array}{ccccc}
\left\langle\hat{n}_{\vec{k}} \hat{n}_{-\vec{k}}\right\rangle_{0} & 0 & 0 & 0 & 0 \\
0 & \left\langle\hat{\vec{J}}_{\vec{k}} \cdot \hat{\vec{\jmath}}_{-\vec{k}}\right\rangle_{0} & 0 & 0 & 0 \\
0 & 0 & \left\langle\hat{h}_{\vec{k}} \hat{h}_{-\vec{k}}\right\rangle_{0} & 0 & 0 \\
0 & 0 & 0 & \left\langle\hat{\pi}_{\vec{k}}: \hat{\leftrightarrow}_{-\vec{k}}\right\rangle_{0} & 0 \\
0 & 0 & 0 & 0 & \left\langle\hat{\vec{q}}_{\vec{k}} \cdot \hat{\vec{q}}_{-\vec{k}}\right\rangle_{0}
\end{array}\right) ;
$$

$\left(\widetilde{\Phi}^{\mathrm{G}}\right)_{l m}^{-1}(\vec{k})$ - элементы матрицы $\left(\widetilde{\Phi}^{\mathrm{G}}\right)^{-1}(\vec{k})$, обратной $\mathrm{\kappa}\left(\widetilde{\Phi}^{\mathrm{G}}\right)(\vec{k}) ; I_{l}^{\mathrm{GH}}(\vec{k})=\left\{I_{\pi}^{\mathrm{GH}}(\vec{k})=\right.$ $\left.\left(1-P_{\mathrm{GH}}\right) i L_{N} \hat{\leftrightarrow}_{\vec{k}}, I_{q}^{\mathrm{GH}}(\vec{k})=\left(1-P_{\mathrm{GH}}\right) i L_{N} \hat{\vec{q}}_{\vec{k}}\right\}$ - обобщенные потоки, в структуру которых входит проекционный оператор Мори расширенного набора динамических переменных $P_{\mathrm{GH}} \widehat{A}=\sum_{l m}\left\langle\widehat{A} \widehat{Y}_{l,-\vec{k}}\right\rangle_{0}\left(\widetilde{\Phi}^{\mathrm{G}}\right)_{l m}^{-1}(\vec{k}) \widehat{Y}_{m, \vec{k}}$ со свойствами: $P_{\mathrm{GH}} P_{\mathrm{GH}}=P_{\mathrm{GH}}$, $P_{\mathrm{GH}}\left(1-P_{\mathrm{GH}}\right)=0, P_{\mathrm{GH}} \widehat{Y}_{l, \vec{k}}=\widehat{Y}_{l, \vec{k}} ; T_{0}^{\mathrm{GH}}\left(t, t^{\prime}\right)$ - оператор эволюции с проекционным оператором $P_{\mathrm{GH}}$. С помощью неравновесного проекционного оператора (13) получаются уравнения обобщенной гидродинамики для простых жидкостей следующей структуры:

$$
\frac{\partial}{\partial t}\left\langle\widehat{Y}_{l, \vec{k}}\right\rangle^{t}-\sum_{m} i \Omega_{l m}^{\mathrm{GH}}(\vec{k})\left\langle\widehat{Y}_{m, \vec{k}}\right\rangle^{t}+\sum_{m} \int_{-\infty}^{t} e^{\varepsilon\left(t^{\prime}-t\right)} \varphi_{l m}^{\mathrm{GH}}\left(\vec{k}, t, t^{\prime}\right)\left\langle\widehat{Y}_{m, \vec{k}}\right\rangle^{t^{\prime}} d t^{\prime}=0,
$$

где $i \Omega_{l m}^{\mathrm{GH}}(\vec{k})$ - элементы матрицы

$$
i \widetilde{\Omega}^{\mathrm{GH}}(\vec{k})=\left(\begin{array}{ccccc}
0 & i \Omega_{n j} & 0 & 0 & 0 \\
i \Omega_{j n} & 0 & i \Omega_{j h} & i \Omega_{j \pi} & 0 \\
0 & i \Omega_{h j} & 0 & 0 & 0 \\
0 & i \Omega_{\pi j} & 0 & 0 & i \Omega_{\pi q} \\
0 & 0 & 0 & i \Omega_{q \pi} & 0
\end{array}\right)_{(\vec{k})}
$$

в структуру которой входят нормированные равновесные корреляционные функции (3)-(6) и новые нормированные равновесные корреляционные функции

$$
\begin{aligned}
& i \Omega_{\pi j}(\vec{k})=\left\langle\hat{\leftrightarrow}_{\vec{k}}: \hat{\vec{\jmath}}_{-\vec{k}}\right\rangle_{0}\left\langle\hat{\vec{j}}_{\vec{k}} \cdot \hat{\vec{\jmath}}_{-\vec{k}}\right\rangle_{0}^{-1}, \\
& i \Omega_{\pi q}(\vec{k})=\left\langle\hat{\leftrightarrow}_{\vec{k}}: \hat{\vec{q}}_{-\vec{k}}\right\rangle_{0}\left\langle\hat{\vec{q}}_{\vec{k}} \cdot \hat{\vec{q}}_{-\vec{k}}\right\rangle_{0}^{-1} ;
\end{aligned}
$$

$\widetilde{\varphi}^{\mathrm{GH}}\left(\vec{k}, t, t^{\prime}\right)$ - обобщенные ядра переноса, которые описывают диссипативные процессы и составляют матрицу функций памяти обобщенной гидродинамики простой жидкости:

$$
\widetilde{\varphi}^{\mathrm{GH}}\left(\vec{k}, t, t^{\prime}\right)=\left(\begin{array}{ccccc}
0 & 0 & 0 & 0 & 0 \\
0 & 0 & 0 & 0 & 0 \\
0 & 0 & 0 & 0 & 0 \\
0 & 0 & 0 & \varphi_{\pi \pi} & \varphi_{\pi q} \\
0 & 0 & 0 & \varphi_{q \pi} & \varphi_{q q}
\end{array}\right)_{\left(\vec{k}, t, t^{\prime}\right)}
$$


Они имеют структуру, аналогичную (8), но построены на обобщенных потоках $I_{\pi}^{\mathrm{GH}}(\vec{k}), I_{q}^{\mathrm{GH}}(\vec{k})$ с оператором эволюции $T_{0}^{\mathrm{GH}}\left(t, t^{\prime}\right)$. Уравнения обобщенной гидродинамики, как и уравнения молекулярной гидродинамики $(1)$, определяют соответствующие временны́е корреляционные функции расширенного набора динамических переменных $\widehat{Y}_{l, \vec{k}}(t)$ :

$$
\widetilde{\Phi}^{\mathrm{GH}}(\vec{k}, t)=\left(\begin{array}{lllll}
\Phi_{n n} & \Phi_{n j} & \Phi_{n h} & \Phi_{n \pi} & \Phi_{n q} \\
\Phi_{j n} & \Phi_{j j} & \Phi_{j h} & \Phi_{j \pi} & \Phi_{j q} \\
\Phi_{h n} & \Phi_{h j} & \Phi_{h h} & \Phi_{h \pi} & \Phi_{h q} \\
\Phi_{\pi n} & \Phi_{\pi j} & \Phi_{\pi h} & \Phi_{\pi \pi} & \Phi_{\pi q} \\
\Phi_{q n} & \Phi_{q j} & \Phi_{q h} & \Phi_{q \pi} & \Phi_{q q}
\end{array}\right)_{(\vec{k}, t)},
$$

причем временнь́е корреляционные функции $\Phi_{\pi \pi}(\vec{k}, t), \Phi_{\pi q}(\vec{k}, t), \Phi_{q \pi}(\vec{k}, t), \Phi_{q q}(\vec{k}, t)$ могут быть связаны с обобщенными коэффициентами (10), т.е. система уравнений

$$
\frac{\partial}{\partial t} \widetilde{\Phi}^{\mathrm{GH}}(\vec{k}, t)-i \widetilde{\Omega}^{\mathrm{GH}}(\vec{k}) \widetilde{\Phi}^{\mathrm{GH}}(\vec{k}, t)+\int_{-\infty}^{t} e^{\varepsilon\left(t^{\prime}-t\right)} \widetilde{\varphi}^{\mathrm{GH}}\left(\vec{k}, t, t^{\prime}\right) \widetilde{\Phi}^{\mathrm{GH}}\left(\vec{k}, t^{\prime}\right) d t^{\prime}=0
$$

дает возможность в единой схеме исследовать временны́е корреляционные функции гидродинамических переменных $\hat{a}_{l, \vec{k}}$, а также обобщенные коэффициенты переноса через корреляционные функции матрицы $i \widetilde{\Omega}^{\mathrm{GH}}(\vec{k})(16)$ и обобщенные ядра переноса матрицы $\widetilde{\phi}^{\mathrm{GH}}\left(\vec{k}, t, t^{\prime}\right)(17)$. При этом в марковском приближении по времени в уравнении $(19)$ в гидродинамическом пределе из $\operatorname{det}\left|i \widetilde{\Omega}^{\mathrm{GH}}(\vec{k})-\widetilde{\Phi}^{\mathrm{GH}}(\vec{k})\right|=0$ находится спектр коллективных возбуждений, который включает тепловую моду $z_{\mathrm{H}}(k)=$ $D_{\mathrm{T}} k^{2}+O\left(k^{4}\right)$, две комплексно-сопряженные звуковые моды $z_{\mathrm{H}}(k)= \pm i \omega_{\mathrm{s}}(k)+z_{\mathrm{s}}(k)$, где $\omega_{\mathrm{s}}(k)=c k+O\left(k^{3}\right)$ - частота распространения звука, $z_{\mathrm{s}}(k)$ - частота затухания звука с коэффициентом затухания Г. Кроме гидродинамических мод, существуют две кинетические моды: $z_{\pi}(k)=\varphi_{\pi \pi}(0)+O\left(k^{2}\right), z_{q}(k)=\varphi_{q q}(0)+O\left(k^{2}\right)$, которые в пределе $k \rightarrow 0$ не исчезают. Здесь

$$
D_{\mathrm{T}}=\frac{v_{\mathrm{T}} q^{2}}{\gamma \varphi_{q q}(0)}=\frac{\lambda}{m n c_{p}}, \quad v_{\mathrm{T}} q^{2}=\frac{m \Phi_{q q}-h^{2}}{n c_{v}},
$$

$D_{\mathrm{T}}$ - коэффициент термодиффузии, $\gamma=c_{p} / c_{v}, c_{p}, c_{v}$ соответственно термодинамические значения теплоемкостей при постоянных давлении и объеме, $\lambda$ - коэффициент теплопроводности, $h$ - термодинамическое значение энтальпии, $c=$ $\gamma /\left(m n S_{2}(k=0)\right)$ - адиабатическая скорость звука, $S_{2}(k=0)$ - статический структурный фактор при $k=0$,

$$
\Gamma=\frac{1}{2}(\gamma-1) D_{\mathrm{T}}+\frac{1}{2} \eta^{\prime \prime}
$$

где

$$
\eta^{\prime \prime}=\frac{v_{p \pi}^{2}}{\varphi_{\pi \pi}(0)}=\frac{(3 / 4) \eta+\kappa}{n m}, \quad v_{p \pi}^{2}=\frac{m S_{2}(0) \Phi_{\pi \pi}(0)-\gamma}{m n S_{2}(0)},
$$

$\eta, \kappa$ - коэффициенты сдвиговой и объемной вязкости. Как видим, $D_{\mathrm{T}}$ и $\eta^{\prime \prime}$ определяются ядрами переноса $\phi_{q q}(0), \phi_{\pi \pi}(0)$ и корреляционными функциями $\Phi_{q q}, \Phi_{\pi \pi}$ 
в соответствующих приближениях. Тепловая мода и две звуковые совпадают с коллективными возбуждениями молекулярной гидродинамики на базе уравнений (1), однако коэффициенты термодиффузии и вязкости определяются функциями памяти высшего порядка $\phi_{q q}, \phi_{\pi \pi}$. Важно отметить, что состоянию молекулярной гидродинамики простой жидкости соответствует неравновесный статистический оператор (12) или при расширенном описании - оператор (13). С одной стороны, неравновесный статистический оператор дает возможность получить соответствующие уравнения переноса для средних значений параметров сокращенного описания, а с другой - согласовать их с соотношениями неравновесной термодинамики, определив энтропию состояния.

Интересным является вопрос о релаксации неравновесного состояния системы взаимодействующих частиц к состоянию молекулярной гидродинамики. Данную задачу рассмотрим в приближении Маркова для функций памяти (7):

$$
\varphi_{m l}^{\mathrm{H}}\left(\vec{k} ; t, t^{\prime}\right)=\varphi_{m l}^{\mathrm{H}}(\vec{k}) \delta\left(t-t^{\prime}\right) .
$$

Тогда уравнения переноса (1) приобретают вид

$$
\frac{\partial}{\partial t}\left\langle\hat{a}_{l, \vec{k}}\right\rangle^{t}-\sum_{m} \Sigma_{l m}^{\mathrm{H}}(\vec{k})\left\langle\hat{a}_{m, \vec{k}}\right\rangle^{t}=0
$$

где

$$
\begin{aligned}
\Sigma_{m l}^{\mathrm{H}}(\vec{k}) & =i \Omega_{m l}^{\mathrm{H}}(\vec{k})-\varphi_{m l}^{\mathrm{H}}(\vec{k}), \\
\varphi_{m l}^{\mathrm{H}}(\vec{k}) & =\int_{0}^{\infty} \varphi_{m l}^{\mathrm{H}}(\vec{k} ; t) d t .
\end{aligned}
$$

В представлении Лапласа по времени уравнения (21) записываются как

$$
z\left\langle\hat{a}_{l, \vec{k}}\right\rangle_{z}-\sum_{m} \Sigma_{l m}^{\mathrm{H}}(\vec{k})\left\langle\hat{a}_{m, \vec{k}}\right\rangle_{z}=-\left\langle\hat{a}_{l, \vec{k}}(t=0)\right\rangle,
$$

откуда можно получить лаплас-изображение для параметров сокращенного описания $\left\langle\hat{a}_{l, \vec{k}}\right\rangle_{z}$ :

$$
\left\langle\hat{a}_{l, \vec{k}}\right\rangle_{z}=-\sum_{m}\left[z I-\Sigma^{\mathrm{H}}(\vec{k})\right]_{l m}^{-1}\left\langle\hat{a}_{m, \vec{k}}(t=0)\right\rangle .
$$

Этому неравновесному состоянию системы отвечает неравновесный статистический оператор обобщенной молекулярной гидродинамики в приближении Маркова:

$$
\varrho^{\mathrm{H}}\left(x^{N} ; t\right)=\varrho_{0}\left(x^{N}\right)\left\{1+\sum_{l, m} \sum_{\vec{k}}\left\langle\hat{a}_{l, \vec{k}}\right\rangle^{t} \widetilde{\Phi}_{l m}^{-1}(\vec{k})\left(\hat{a}_{m,-\vec{k}}-I_{m}^{\mathrm{H}}(-\vec{k} ; t)\right)\right\},
$$

где

$$
I_{l}^{\mathrm{H}}(\vec{k} ; t)=\int_{-\infty}^{t} e^{\varepsilon\left(t^{\prime}-t\right)} T_{0}^{\mathrm{H}}\left(t, t^{\prime}\right) I_{l}^{\mathrm{H}}(\vec{k}) d t^{\prime} .
$$

Здесь следует отметить важную особенность: обозначение (27) является символическим, поскольку предел $\varepsilon \rightarrow+0$ выполняется после термодинамического предела $N \rightarrow \infty, V \rightarrow \infty, N / V=$ const при выполнении соответствующих усреднений

4 Теоретическая и математическая физика, т. 154, № 1, 2008 г. 
с неравновесным статистическим оператором (26) при получении уравнений переноса (1) или (21) в марковском приближении.

Теперь поставим задачу нахождения неравновесного статистического оператора из уравнения Лиувилля. Использовав метод неравновесного статистического оператора [13]-[15] и граничное условие, утверждающее, что $\varrho\left(x^{N} ; t\right)$ при $t=t_{0}$ равно $\varrho^{\mathrm{H}}\left(x^{N} ; t_{0}\right)$, получим неравновесный статистический оператор системы, неравновесное состояние которой релаксирует к состоянию молекулярной гидродинамики:

$$
\varrho\left(x^{N} ; t\right)=\varrho^{\mathrm{H}}\left(x^{N} ; t\right)-\int_{-\infty}^{t} e^{\varepsilon\left(t^{\prime}-t\right)} T_{q}\left(t, t^{\prime}\right)\left(1-P_{q}\left(t^{\prime}\right)\right) i L_{N} \varrho^{\mathrm{H}}\left(x^{N} ; t^{\prime}\right) d t^{\prime},
$$

в котором проекционный оператор Кавасаки-Гантона имеет следующую структуру:

$$
\begin{aligned}
P_{q} \varrho^{\prime}\left(x^{N} ; t\right)=( & \left.\varrho^{\mathrm{H}}\left(x^{N} ; t\right)-\sum_{l} \frac{\delta \varrho^{\mathrm{H}}\left(x^{N} ; t\right)}{\delta\left\langle\hat{a}_{l, \vec{k}}\right\rangle^{t}}\left\langle\hat{a}_{l, \vec{k}}\right\rangle^{t}\right) \int \varrho^{\prime}\left(x^{N} ; t\right) d \Gamma_{N}+ \\
& +\sum_{l} \frac{\delta \varrho^{\mathrm{H}}\left(x^{N} ; t\right)}{\delta\left\langle\hat{a}_{l, \vec{k}}\right\rangle^{t}} \int \hat{a}_{l, \vec{k}} \varrho^{\prime}\left(x^{N} ; t\right) d \Gamma_{N}
\end{aligned}
$$

и $T_{q}\left(t, t^{\prime}\right)$ - оператор эволюции, но с проекционным оператором (29). Далее, раскрывая действие оператора (29) и оператора Лиувилля на $\varrho^{\mathrm{H}}\left(x^{N} ; t^{\prime}\right)$, получим

$$
\begin{aligned}
& \left(1-P_{q}(t)\right) i L_{N} \varrho^{\mathrm{H}}\left(x^{N} ; t\right)= \\
& \quad=\varrho_{0}\left(x^{N}\right) \sum_{l, m} \sum_{\vec{k}}\left(1-\bar{P}_{\mathrm{H}}(t)\right)\left(\dot{\hat{a}}_{l,-\vec{k}}-\delta \dot{I}_{l}^{\mathrm{H}}(-\vec{k} ; t)\right) \widetilde{\Phi}_{l m}^{-1}(\vec{k})\left\langle\hat{a}_{m, \vec{k}}\right\rangle^{t},
\end{aligned}
$$

где $\dot{\hat{a}}_{m, \vec{k}}=i L_{N} \hat{a}_{m, \vec{k}}, \dot{I}_{m}^{\mathrm{H}}(\vec{k} ; t)=i L_{N} I_{m}^{\mathrm{H}}(\vec{k} ; t)$,

$$
\bar{P}_{\mathrm{H}}(t) \ldots=\sum_{l, m}\left\langle\ldots \hat{a}_{l,-\vec{k}}\right\rangle_{0} \widetilde{\Phi}_{l m}^{-1}(\vec{k})\left(\hat{a}_{m, \vec{k}}-I_{m}^{\mathrm{H}}(\vec{k} ; t)\right)
$$

- "проекционный" оператор, $\delta \dot{I}_{l}^{\mathrm{H}}(\vec{k} ; t)=\dot{I}_{l}^{\mathrm{H}}(\vec{k} ; t)-\left\langle\dot{I}_{l}^{\mathrm{H}}(\vec{k} ; t)\right\rangle$ с учетом соотношения $\left\langle\dot{\hat{a}}_{m, \vec{k}}\right\rangle_{0}=0$. Учитывая выражение (30), неравновесный статистический оператор (28) можно записать в виде

$$
\begin{aligned}
& \varrho\left(x^{N} ; t\right)=\left(1+\sum_{l, m} \sum_{\vec{k}}\left(\left\langle\hat{a}_{l, \vec{k}}\right\rangle^{t} \widetilde{\Phi}_{l m}^{-1}(\vec{k})\left(\hat{a}_{m,-\vec{k}}-I_{m}^{\mathrm{H}}(-\vec{k} ; t)\right)-\right.\right. \\
& \quad-\int_{-\infty}^{t} e^{\varepsilon\left(t^{\prime}-t\right)} T_{q}\left(t, t^{\prime}\right)\left(1-\bar{P}_{\mathrm{H}}\left(t^{\prime}\right)\right) \times \\
& \left.\left.\quad \times\left(\dot{\hat{a}}_{l,-\vec{k}}-\delta \dot{I}_{l}^{\mathrm{H}}\left(-\vec{k} ; t^{\prime}\right)\right) \widetilde{\Phi}_{l m}^{-1}(\vec{k})\left\langle\hat{a}_{m, \vec{k}}\right\rangle^{t^{\prime}} d t^{\prime}\right)\right) \varrho_{0}\left(x^{N}\right) .
\end{aligned}
$$

В отличие от неравновесного статистического оператора молекулярной гидродинамики (12) оператор (32) является функцией не только гидродинамических переменных $\hat{a}_{m, \vec{k}}$ и их обобщенных потоков $I_{m}^{\mathrm{H}}(\vec{k} ; t)$, но и функцией новых переменных, которые получаются при действии оператором Лиувилля на гидродинамические переменные и их обобщенные потоки. С помощью этого неравновесного статистического 
оператора, используя соотношение

$$
\frac{\partial}{\partial t}\left\langle\hat{a}_{l, \vec{k}}\right\rangle^{t}=\left\langle\dot{\hat{a}}_{l, \vec{k}}\right\rangle^{t}
$$

можно получить обобщенные уравнения переноса

$$
\frac{\partial}{\partial t}\left\langle\hat{a}_{l, \vec{k}}\right\rangle^{t}=\left\langle\dot{\hat{a}}_{l, \vec{k}}\right\rangle_{\mathrm{H}}^{t}-\sum_{m} \int_{-\infty}^{t} e^{\varepsilon\left(t^{\prime}-t\right)} \varphi_{l m}\left(\vec{k} ; t, t^{\prime}\right)\left\langle\hat{a}_{m, \vec{k}}\right\rangle^{t^{\prime}} d t^{\prime}
$$

с функциями памяти

$$
\begin{aligned}
\varphi_{l m}\left(\vec{k} ; t, t^{\prime}\right)= & \sum_{m^{\prime}}\left\langle\dot{\hat{a}}_{l, \vec{k}} T_{q}\left(t, t^{\prime}\right)\left(1-\bar{P}_{\mathrm{H}}\left(t^{\prime}\right)\right)\left(\dot{\hat{a}}_{m^{\prime},-\vec{k}}-\delta \dot{I}_{m^{\prime}}^{\mathrm{H}}\left(-\vec{k} ; t^{\prime}\right)\right)\right\rangle_{0} \widetilde{\Phi}_{m^{\prime} m}^{-1}(\vec{k}), \\
\left\langle\dot{\hat{a}}_{l, \vec{k}}\right\rangle_{\mathrm{H}}^{t}= & \sum_{m} i \Omega_{l m}^{\mathrm{H}}(\vec{k})\left\langle\hat{a}_{m, \vec{k}}\right\rangle^{t}-\sum_{m} \varphi_{l m}^{\mathrm{H}}(\vec{k})\left\langle\hat{a}_{m, \vec{k}}\right\rangle^{t}+ \\
& +\sum_{m, m^{\prime}} i \Omega_{l m^{\prime}}^{\mathrm{H}}(\vec{k}) D_{m^{\prime} m}(\vec{k})\left\langle\hat{a}_{m, \vec{k}}\right\rangle^{t},
\end{aligned}
$$

где $D_{m^{\prime} m}(\vec{k})=\sum_{l^{\prime}}\left\langle\dot{\hat{a}}_{m^{\prime}, \vec{k}} I_{l^{\prime}}^{\mathrm{H}}\left(-\vec{k} ; t^{\prime}\right)\right\rangle_{0} \widetilde{\Phi}_{l^{\prime} m}^{-1}(\vec{k})$. Как можно видеть из структуры уравнения (33), первым слагаемым в правой части является вклад молекулярной гидродинамики, для которого хорошо изучены коллективные возбуждения. Функции памяти (34) имеют сложную структуру. Раскрывая действие оператора $\left(1-\bar{P}_{\mathrm{H}}(t)\right)$ на $\dot{\hat{a}}_{m, \vec{k}}$ и $\delta \dot{I}_{m}^{\mathrm{H}}\left(\vec{k} ; t^{\prime}\right)$, приходим к

$$
\begin{aligned}
\left(1-\bar{P}_{\mathrm{H}}(t)\right) \dot{\hat{a}}_{m, \vec{k}} & =\dot{\hat{a}}_{m, \vec{k}}-\sum_{m^{\prime}} i \Omega_{m m^{\prime}}^{\mathrm{H}}(\vec{k})\left(\hat{a}_{m^{\prime}, \vec{k}}-I_{m^{\prime}}^{\mathrm{H}}(\vec{k} ; t)\right), \\
\left(1-\bar{P}_{\mathrm{H}}(t)\right) \delta \dot{I}_{m}^{\mathrm{H}}(\vec{k} ; t) & =\dot{I}_{m}^{\mathrm{H}}(\vec{k} ; t)-\left\langle\dot{I}_{m}^{\mathrm{H}}(\vec{k} ; t)\right\rangle_{0}+\sum_{m^{\prime}} \varphi_{m m^{\prime}}^{\mathrm{H}}(\vec{k})\left(\hat{a}_{m^{\prime}, \vec{k}}-I_{m^{\prime}}^{\mathrm{H}}(\vec{k} ; t)\right) ;
\end{aligned}
$$

для $\varphi_{l m}\left(t, t^{\prime}\right)$ получаем

$$
\begin{aligned}
\varphi_{l m}\left(\vec{k} ; t, t^{\prime}\right)= & \sum_{m^{\prime}}\left(\left\langle\dot{\hat{a}}_{l, \vec{k}} T_{q}\left(t, t^{\prime}\right) \dot{\hat{a}}_{m^{\prime},-\vec{k}}\right\rangle_{0}-\left\langle\dot{\hat{a}}_{l, \vec{k}} T_{q}\left(t, t^{\prime}\right) \dot{I}_{m^{\prime}}^{\mathrm{H}}\left(-\vec{k} ; t^{\prime}\right)\right\rangle_{0}\right) \widetilde{\Phi}_{m^{\prime} m}^{-1}(\vec{k})- \\
- & \sum_{m^{\prime}, l^{\prime}}\left(\left\langle\dot{\hat{a}}_{l, \vec{k}} T_{q}\left(t, t^{\prime}\right) \hat{a}_{m^{\prime},-\vec{k}}\right\rangle_{0}-\left\langle\dot{\hat{a}}_{l, \vec{k}} T_{q}\left(t, t^{\prime}\right) I_{m^{\prime}}^{\mathrm{H}}\left(-\vec{k} ; t^{\prime}\right)\right\rangle_{0}\right) \times \\
& \times\left(i \Omega_{m^{\prime} l^{\prime}}^{\mathrm{H}}(\vec{k})-\varphi_{m^{\prime} l^{\prime}}^{\mathrm{H}}(\vec{k})\right) \widetilde{\Phi}_{l^{\prime} m}^{-1}(\vec{k}) .
\end{aligned}
$$

Уравнения переноса (33) по структуре функций памяти $\varphi_{l m}\left(\vec{k} ; t, t^{\prime}\right)$, с учетом $\varphi_{m m^{\prime}}^{\mathrm{H}}(\vec{k}) i \Omega_{m m^{\prime}}^{\mathrm{H}}(\vec{k})$, содержат второй порядок по временны́м корреляционным функциям. Вместе с неравновесным статистическим оператором они могут описывать неравновесные процессы, связанные с релаксацией состояния системы взаимодействующих частиц к состоянию молекулярной гидродинамики. Важными являются уравнения для временны́х корреляционных функций, отвечающие системе уравнений переноса (33). Согласно структуре функций памяти (38) они должны строиться для расширенного набора динамических переменных $\widehat{Y}_{l, \vec{k}}$, как и в случае неравновесного статистического оператора (13). Эти вопросы будут рассмотрены в следующих работах. 


\section{3. ЗАКЛЮЧЕНИЕ}

Таким образом, в этой работе мы изложили основные уравнения молекулярной гидродинамики и их обобщения, которые получены с помощью соответствующих неравновесных статистических операторов метода Зубарева. При таком подходе проекционные операторы Кавасаки-Гантона и Мори возникают естественно, а явный вид неравновесного статистического оператора дает возможность согласовать основные уравнения переноса с соотношениями неравновесной термодинамики. Важным является тот факт, что состоянию молекулярной гидродинамики в методе Зубарева соответствует определенный неравновесный статистический оператор. В настоящее время характеристики (коллективные возбуждения, временны́е корреляционные функции, коэффициенты переноса) состояния молекулярной гидродинамики для жидкостей широко изучены, что может служить основой выхода за рамки молекулярной гидродинамики. Мы попытались кратко реализовать такой подход с помощью метода неравновесного статистического оператора Зубарева, рассмотрев релаксацию неравновесного состояния системы к состоянию молекулярной гидродинамики с ядрами переноса в приближении Маркова, которое для простой жидкости Леннарда-Джонса хорошо исследовано [1], [8], [19].

\section{Список литературы}

[1] J.P. Boon, S. Yip, Molecular Hydrodynamics, McGraw-Hill, New York, 1980.

[2] R. D. Mountain, Adv. Mol. Relax. Proc., 9 (1977), 225.

[3] С. Лавси, Т. Шпрингер (ред.), Динамические свойства твердых тел и жидкостей. Исследования методом рассеяния нейтронов, Мир, М., 1980.

[4] П. Резибуа, М. Де Ленер, Классическая кинетическая теория жидкостей и газов, Мир, М., 1980.

[5] L. P. Kadanoff, P. C. Martin, Ann. Phys., 24 (1963), 419.

[6] М. В. Сергеев, ТМФ, 21:3 (1974), 402.

[7] С. В. Тищенко, ТМФ, 26:1 (1976), 96.

[8] I. M. de Schepper, E. G. D. Cohen, C. Bruin, J. C. van Rijs, W. Montrooij, L. A. de Graaf, Phys. Rev. A, 38:1 (1988), 271.

[9] Ю. Ф. Церковников, ТМФ, 63:3 (1985), 440.

[10] И. М. Мрыглод, М. В. Токарчук, K статистической гидродинамике простых жидкостей. Обобщенные коэффициенты переноса, Препринт АН УРСР, ИФКС, 91-6У, Киев, 1991.

[11] И. М. Мрыглод, М. В. Токарчук, ВАНТ, 1992, № 3(24), 134.

[12] I. M. Mryglod, I. P. Omelyan, M. V. Tokarchuk, Mol. Phys., 84:2 (1995), 235.

[13] Д. Н. Зубарев, Неравновесная статистическая термодинамика, Наука, М., 1971.

[14] Д.Н. Зубарев, "Современные методы статистической теории неравновесных процессов", Итоги науки и техники. Соврем. проблемы матем., 15, ВИНИТИ, М., 1980, 131.

[15] Д. Н. Зубарев, В. Г. Морозов, Г. Рёпке, Статистическая механика неравновесных процессов, Т. 2, Физматлит, М., 2002.

[16] Д. Н. Зубарев, М. В. Токарчук, ТМФ, 70:2 (1987), 234.

[17] A. Z. Akcasu, E. Deniels, Phys. Rev. A, 2:3 (1970), 962.

[18] И. М. Мрыглод, Статистическая теория коллективных возбуждений в жидкостях: подход обобщенных коллективных мод, Автореф. дисс. ... докт. физ.-матем. наук, Львов. нац. ун-т им. И. Франко, Львов, 2000.

[19] I. P. Omelyan, I. M. Mryglod, Condens. Matter Phys., 1994, № 4, 128.

[20] I. M. Mryglod, I. P. Omelyan, Phys. Lett. A, 205:5-6 (1995), 401. 
[21] I. M. Mryglod, M. V. Tokarchuk, R. Folk, Phys. A, 220:3-4 (1995), 325.

[22] I. M. Mryglod, R. Folk, Phys. A, 234:1-2 (1996), 129.

[23] И. М. Мрыглод, М. В. Токарчук, ТМФ, 115:1 (1998), 132.

[24] И. М. Мрыглод, Ю. К. Рудавський, М. В. Токарчук, О. Ф. Бацевич, Укр. физ. журн., 44:8 (1999), 1030.

[25] И. М. Мрыглод, Ю.К. Рудавський, М. В. Токарчук, О.Ф. Бацевич, Укр. физ. журн., 44:9 (1999), 1174.

[26] I. Mryglod, R. Folk, S. Dubyk, Yu. Rudavskii, Condens. Matter Phys., 2:2(18) (1999), 221.

[27] I. P. Omelyan, I. M. Mryglod, M. V. Tokarchuk, Condens. Matter Phys., 1:1(13) (1998), 179.

[28] I. P. Omelyan, I. M. Mryglod, M. V. Tokarchuk, Phys. Rev. E, 57:6 (1998), 6667.

[29] И. П. Омелян, Р. И. ЖКелем, М. В. Токарчук, Укр. физ. журн., $42: 6$ (1997), 684.

[30] I. P. Omelyn, Mol. Phys., 93:1 (1998), 123.

[31] I. P. Omelyan, M. V. Tokarchuk, J. Phys. Condens. Matter, 12:30 (2000), L505.

[32] I. P. Omelyan, I. M. Mryglod, M. V. Tokarchuk, Condens. Matter Phys., 8:1(41) (2005), 25.

[33] T. Bryk, I. Mryglod, J. Phys. Condens. Matter, 16:41 (2004), L463.

[34] T. Bryk, I. Mryglod, Condens. Matter Phys., 6:3(35) (2003), 395.

[35] T. Bryk, I. Mryglod, Phys. Rev. B, 71:13 (2005), 132202.

[36] В. В. Игнатюк, И. М. Мрыглод, М. В. Токарчук, ФНТ, 25:5 (1999), 407.

[37] В. В. Игнатюк, И. М. Мрыглод, М.В. Токарчук, ФHT, 25:11 (1999), 1145.

[38] I. M. Mryglod, Condens. Matter Phys., 1997, № 10, 115.

[39] T. M. Bryk, I. M. Mryglod, G. Kahl, Phys. Rev. E, 56:3 (1997), 2903.

[40] T. M. Bryk, I. M. Mryglod, Phys. Lett. A, 261:5-6 (1999), 349.

[41] T. M. Bryk, I. M. Mryglod, Condens. Matter Phys., 2:2(18) (1999), 285.

[42] T. Bryk, I. Mryglod, Condens. Matter Phys., 7:2(38) (2004), 285.

[43] T. Bryk, I. Mryglod, J. Phys. Condens. Matter, 13:7 (2001), 1343.

[44] T. Bryk, I. Mryglod, J. Phys. Studies, 8:1 (2004), 35.

[45] T. Scopigno, U. Balucani, G. Ruocco, F. Sette, Phys. Rev. E, 63:1 (2000), 011210.

[46] A. V. Mokshin, R. M. Yulmetyev, R. M. Khusnutdinoff, P. Hänggi, J. Phys. Condens. Matter, 19:4 (2007), 046209.

[47] Д. Н. Зубарев, В. Г. Морозов, И. П. Омелян, М. В. Токарчук, ТМФ, 87:1 (1991), 113.

[48] Д. Н. Зубарев, В. Г. Морозов, И. П. Омелян, М. В. Токарчук, ТМФ, 96:3 (1993), 325.

[49] M. V. Tokarchuk, I. P. Omelyan, A. E. Kobryn, Condens. Matter Phys., 1:4(16) (1998), 687.

[50] V. G. Morozov, A. E. Kobryn, M. V. Tokarchuk, Condens. Matter Phys., 1994, № 4, 117.

[51] I. P. Omelyan, M. V. Tokarchuk, Phys. A, 234:1-2 (1996), 89.

[52] A. E. Kobryn, I. P. Omelyan, M. V. Tokarchuk, J. Stat. Phys., 92:5-6 (1998), 973.

[53] A. E. Kobryn, V. G. Morozov, I. P. Omelyan, M. V. Tokarchuk, Phys. A, 230:1-2 (1996), 189.

[54] P. A. Hlushak, M. V. Tokarchuk, Condens. Matter Phys., 7:3(39) (2004), 639.

[55] I. M. Mryglod, V. V. Ignatyuk, J. Phys. Studies, 1:2 (1997), 181.

[56] Ю. А. Церковников, ТМФ, 85:1 (1990), 124.

[57] Ю. А. Церковников, ТМФ, 118:1 (1999), 105.

[58] Ю. А. Церковников, ТМФ, 119:1 (1999), 142.

[59] I. M. Mryglod, A. M. Hachkevych, Condens. Matter Phys., 1995, № 5, 105.

[60] Г. О. Балабанян, ТМФ, 82:3 (1990), 450.

[61] Г. О. Балабанян, ТМФ, 85:1 (1990), 102. 